\title{
Synthesis of CdS nanocrystals using polymer metal complex as a single source precursor and their physicochemical properties
}

\author{
Tansir Ahamad* and Saad M Alshehri \\ Department of Chemistry, King Saud University, Riyadh-11451, Saudi Arab. \\ ${ }^{*}$ Corresponding author: Tel.: +966-1-4675971; fax: +966-1-4674018. \\ e-mail: tahamed@ksu.edu.sa
}

Keywords: Chalcogenide, nanocrystals, transmission electron microscopy, CdS, band gap.

\begin{abstract}
Polymeric ligand (TSCF) have been synthesized via polycondansation of thiosemicarbazide with formaldehyde and its polymer metal complex [TSCF-Cd(II)] has been prepared with $\mathrm{Cd}(\mathrm{II})$ ion. Two different batches of $\mathrm{CdS}$ chalcogenide, semiconductor nanocrystals have been synthesized by hydrothermal treatment of TSCF-Cd(II)] using a glass Q tube reactor at $200{ }^{\circ} \mathrm{C}$ and $250{ }^{\circ} \mathrm{C}$, and marked as $(\mathrm{CdS})_{1}$ and $(\mathrm{CdS})_{2}$ respectively. The resultant nanocrystals were characterized by a variety of methods for thier physochemcial properties. The TEM micrographs showed well-defined, close to hexagonal particles, and the lattice fringes in the HRTEM images confirmed their crystalline nature. The sizes of $(\mathrm{CdS})_{1}$ and $(\mathrm{CdS})_{2}$ were 40 and $50 \mathrm{~nm}$ respectively but their morphologies were similar. The optical band gap energies $(2.52 \mathrm{eV} / 2.48 \mathrm{eV})$ and photoluminescence peaks $(532 / 534 \mathrm{~nm})$ of the synthesized CdS nanocrystals suggest that they can be promising photocatalysts. The conductivities and the dielectric constants of the $\mathrm{CdS}$ nanocrystals were also studied and the activation energy value of $(\mathrm{CdS})_{1}$ and $(\mathrm{CdS})_{2}$ nanocrystals were found to be $0.64 \mathrm{eV}$ and $0.70 \mathrm{eV}$.
\end{abstract}




\section{Introduction}

Development of semiconductor nanocrystals has been intensively pursued because of their sizedependent characteristics, and their novel electronic, magnetic, optical, chemical, and mechanical properties that cannot be obtained in their bulk counterparts. Cadmium sulfide (CdS) is one of the most important chalcogenide semiconductor materials because of its direct band gap $(2.42 \mathrm{eV}$ at $300 \mathrm{~K}$ ), which leads to many valuable properties such as photoluminescence, electroluminescence, and photocatalytic effects [1]. Considerable effort has been devoted to prepare monodisperse nanocrystals with controlled size and shape [2,3]. A wide range of approaches for synthesis of CdS nanoparticles has been reported, such as the hydrothermal method, solvothermal methods, low temperature synthesis and liquid ammonia synthesis etc. [4-5]. However, the product obtained in some cases suffers from particle agglomeration and a broad final particle size distribution [6,7]. Xie et al. [6] have reported branch-like CdS micropatterns, using thiosemicarbazide as both a sulfur source and a capping ligand in a methanol/water system. The work is important for understanding the formation of complicated $\mathrm{CdS}$ fractals and their potential applications in microelectronic devices. However, the shape produced was irregular, and the electron diffraction from the CdS architectures showed that the fractals were polycrystalline in nature [6]. When used in combination with capping agents such as oleic acid or oleyl amine, polyvinyl alcohol, polyethylene glycol, etc, this procedure results in the formation of monodisperse nanoparticles with good crystallinity and uniform size [7, 8]. The use of single source precursors for the synthesis of nanoparticles has proven to be efficient routes for the synthesis of high quality nanocrystals. Ligands properties of the metal complexes used as precursor could be used in the modification of the size and shape of the nanoparticles. For example, alkyl-phenyl dithiocarbamate complexes have been used as single source precursors to prepare $\mathrm{ZnS}, \mathrm{CdS}$, and $\mathrm{HgS}$ semiconductor nanoparticles [9]. However to date, there is no published, controlled, research data on synthesis of CdS nanoparticles using polymer metal complexes as single-source precursor. In this study, we have synthesized a polymer metal complex TSCF-Cd(II) as a single source precursor by the reaction of 
thiosemicarbazide- formaldehyde resin with $\mathrm{Cd}(\mathrm{II})$ ion and $\mathrm{CdS}$ nanocrystals have been prepared by hydrothermal treatment of $\mathrm{TSCF}-\mathrm{Cd}(\mathrm{II})$ at $200{ }^{\circ} \mathrm{C}$ and $250{ }^{\circ} \mathrm{C}$ without using any extra capping agent.

\section{Expremental}

$\mathrm{CdCl}_{2} \cdot 6 \mathrm{H}_{2} \mathrm{O}$, thiosemicarbazide, formaldehyde were purchaged from Sigma Aldrich and were of analytical grade. In a $250 \mathrm{~mL}$ round-bottom flask, thiosemicarbazide (9.14 g, $0.1 \mathrm{~mol})$ and 37\% aqueous solution of formaldehyde $(22.5 \mathrm{ml}, 0.3 \mathrm{~mol})$ were mixed in $60 \mathrm{ml} \mathrm{N}, \mathrm{N}$ dimethylformamide (DMF) in basic medium and the mixture was stirred at $60^{\circ} \mathrm{C}$ for $24 \mathrm{~h}$ according previous reported method [10]. The reaction mixture was evaporated using a rotary evaporator, and the final mixture was cooled and precipitated into deionized water. A solid light yellow product was obtained, which was dried in a vacuum oven to remove trapped solvent to give polymeric ligand (TSCF). To prepare cadmium sulfide $(\mathrm{CdS})_{1}$ nanocrystals, $1 \mathrm{mmol}$ of $\mathrm{CdCl}_{2} \cdot 6 \mathrm{H}_{2} \mathrm{O}$ were mixed with $2 \mathrm{mmol}$ of TSCF in a 35-mL Q-tube reactor, containing $15 \mathrm{~mL}$ of distilled water, which forms a yellow color of TSCF$\mathrm{Cd}(\mathrm{II})$. The Q-tube was maintained at $200{ }^{\circ} \mathrm{C}$ for $5 \mathrm{~h}$, the resulting yellow precipitate was washed with distilled water and ethanol, and dried in a vacuum at $60^{\circ} \mathrm{C}$ for $4 \mathrm{~h}$. For the synthesis of $(\mathrm{CdS})_{2}$, the Q-tube temperature was mantained at $250^{\circ} \mathrm{C}$ for $5 \mathrm{~h}$, keeping other conditions the same as for $(\mathrm{CdS})_{1}$. The synthesized nanocrystals were characterized by XRD using a PAN alytical X'Pert Pro X-ray diffractometer with $\mathrm{Cu} \mathrm{K} \alpha$ radiation $(\lambda=1.54 \AA)$. Microstructural analyses of samples used a field emission scanning electron microscope (FESEM-JSM 7600F) and field emission transmission electron microscope (FETEM-JEM 2100F). The UV-vis absorption spectra of were recorded using a Perkin Elmer spectrometer. The photoluminescence spectra were measured on a Perkin Elmer LS-55 fluorescence spectrometer. D.C. conductivity was determined by measuring the resistance of the sample using an electrometer in the V/I mode. Dielectric constant $€$ and ac conductivity ( $\sigma a c)$, of the samples were determined using an impedance analyzer over a frequency range $10 \mathrm{kHz}-13 \mathrm{MHz}$. 


\section{Results and discussion}

CdS nanoparticles have been prepared by hydrothermal treatment of TSCF-Cd(II) at $200{ }^{\circ} \mathrm{C}$ and $250{ }^{\circ} \mathrm{C}$ for $5 \mathrm{~h}$. Fig. 1a and $1 \mathrm{~b}$ shows TEM images of both types of $\mathrm{CdS}$ nanocrystals. The sizes of $(\mathrm{CdS})_{1}$ and $(\mathrm{CdS})_{2}$ were 40 and $50 \mathrm{~nm}$ respectively and their morphologies were similar. The particle size distribution histogram of nanoparticles was obtained and shows that the particle size rages form $20 \mathrm{~nm}$ to $80 \mathrm{~nm}$ in diameter with an average size of $40 \mathrm{~nm}$ and $50 \mathrm{~nm}$ for $(\mathrm{CdS})_{1}$ and $(\mathrm{CdS})_{2}$ respectively. This result also indicates that the particles are monodispersed without aggregation. The HRTEM images of Fig. 1c and 1d show single crystal $(\mathrm{CdS})_{1}$ and $(\mathrm{CdS})_{2}$ displaying clear fringes with interlayer spacing measured to be $0.336 \pm 0.005 \mathrm{~nm}$, very close to the lattice spacing of the (002) plane in hexagonal CdS. The corresponding EDS spectra indicate that the dendrites consist of $\mathrm{Cd}$ and $\mathrm{S}$ with a ratio of $1: 1$. Careful examination of the images shown in Fig. 1e and $1 \mathrm{f}$ indicates the interdigitation of molecular bonds in individual CdS nanocrystals. In this study the crystal structure of CdS is hexagonal; all the Cd-Cd and S-S atomic distances (4.136 $\AA$ with a standard deviation of $\pm 0.1 \AA$ ) are in the normal range for the related structural dimension (along the $\mathrm{a}=\mathrm{b}$ axis) and the length (i.e. the dimension along the $\mathrm{c}$ axis) is $6.723 \pm 0.2 \AA[11]$. 

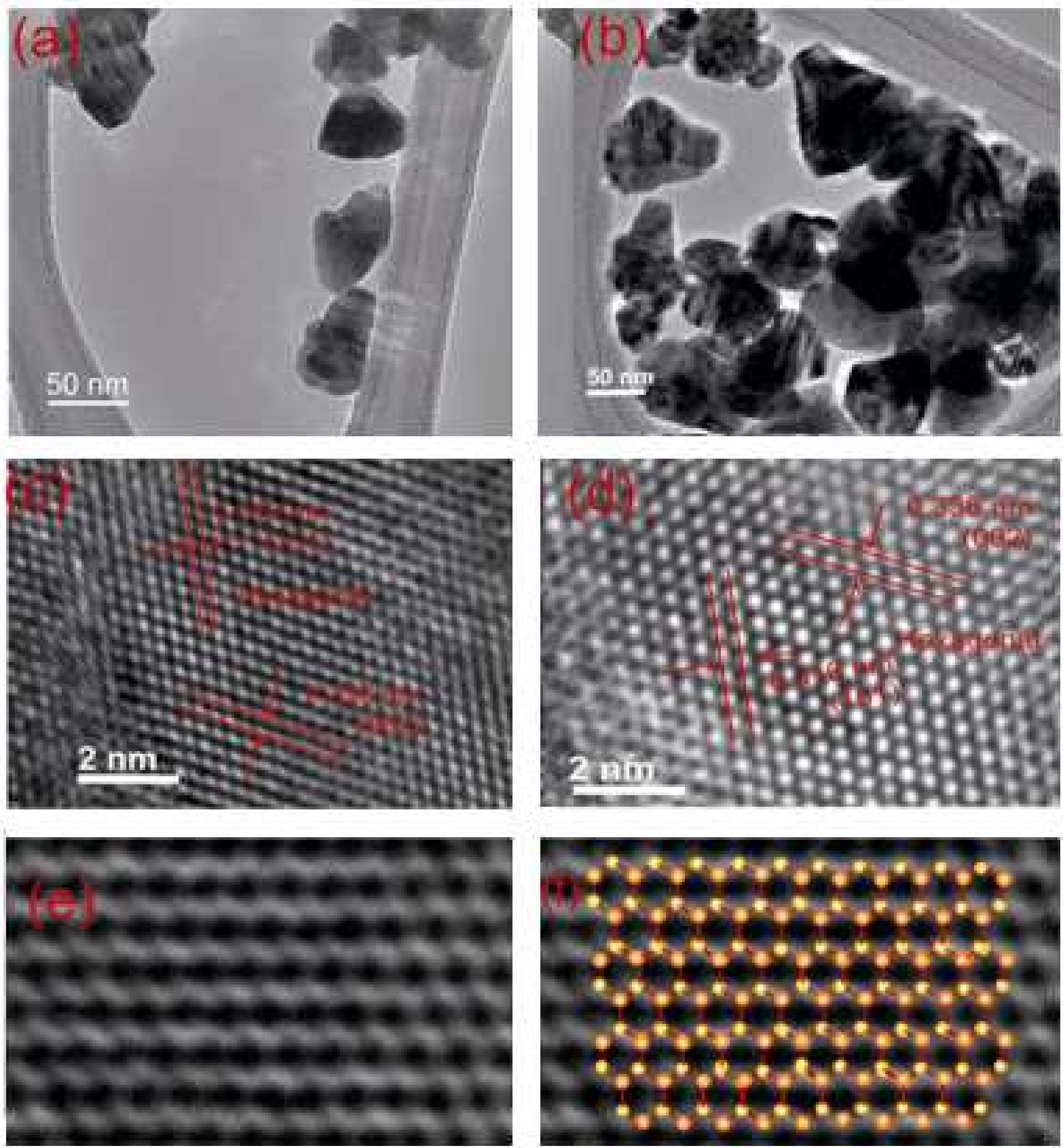

Fig. 1. (a, b) TEM images of CdS nanocrystals prepared at $200^{\circ} \mathrm{C}$ and $250{ }^{\circ} \mathrm{C}$ respectively; (c, d) HRTEM images showing the clear fringes produced by the nanocrystals; and $(e, f)$ interdigitation of molecular bonds. (a, c, and e) are for $(\mathrm{CdS})_{1}$ and $(b, d$, and $\mathrm{f})$ are $(\mathrm{CdS})_{2}$. 

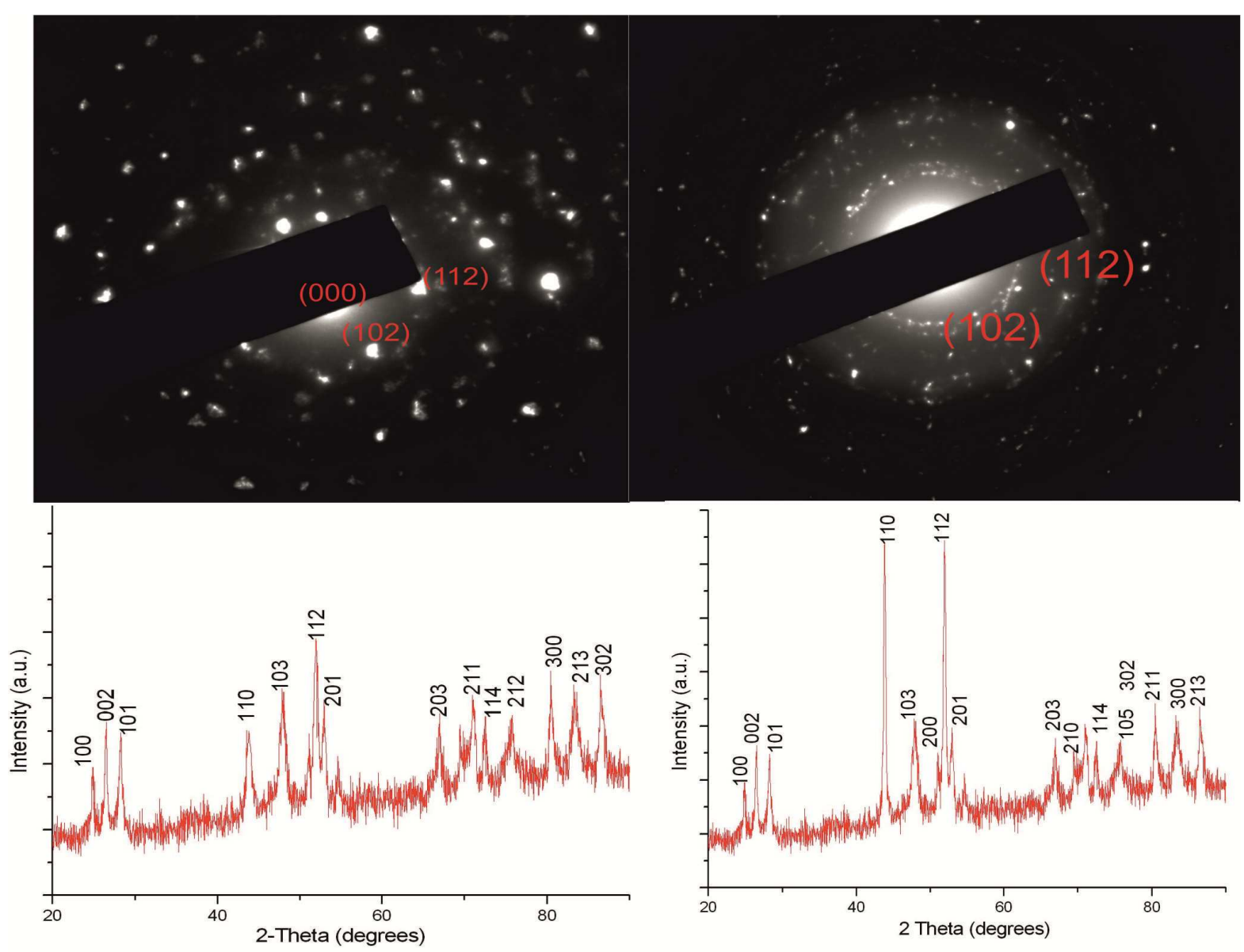

Fig. 2. XRD and SAED patterns of CdS nanocrystals. $(\mathrm{CdS})_{1}$ on left and $(\mathrm{CdS})_{2}$ on right.

Fig. 2 shows the XRD patterns of the as-prepared samples, shows strong and sharp diffraction peaks, indicating that the product was crystalline and the SAED patterns of $(\mathrm{CdS})_{1}$ and $(\mathrm{CdS})_{2}$, indicates the formtion of single-crystalline structure. All the diffraction peaks in XRD can be indexed to pure hexagonal CdS with lattice constants of $a=\mathrm{b}=4.147 \AA$ and $c=6.747 \AA$, which are consistent with the data in the standard card (JCPDS 75-1545). No byproduct peaks were found. The (102), (110), (103), and (112) planes of wurtzite CdS are clearly distinguishable in the pattern [12]. The SAED pattern consists of broad diffuse rings, which are indicative of the small size of the particles. The diffraction rings can be indexed to the (102) and (112) planes, confirming the wurtzite phase [13]. Average particle size of nanocrystal is estimated according to Scherrer equation [14],

$D=\frac{K \lambda}{\beta_{2 \theta} \operatorname{Cos} 26}$ 
Where $\mathrm{K}$ is a constant taken to be $0.94, \lambda$ is the $\mathrm{X}$-ray wavelength and $\beta_{2 \theta}$ is the full width at half maximum of the XRD selected diffraction peak on the $2 \theta$ scale and $\theta$ is diffraction angle. The particle size of the synthesized nanocrystal are found to be $39 \mathrm{~nm}$ and $48 \mathrm{~nm}$ for $(\mathrm{CdS})_{1}$ and $(\mathrm{CdS})_{2}$ respectively.
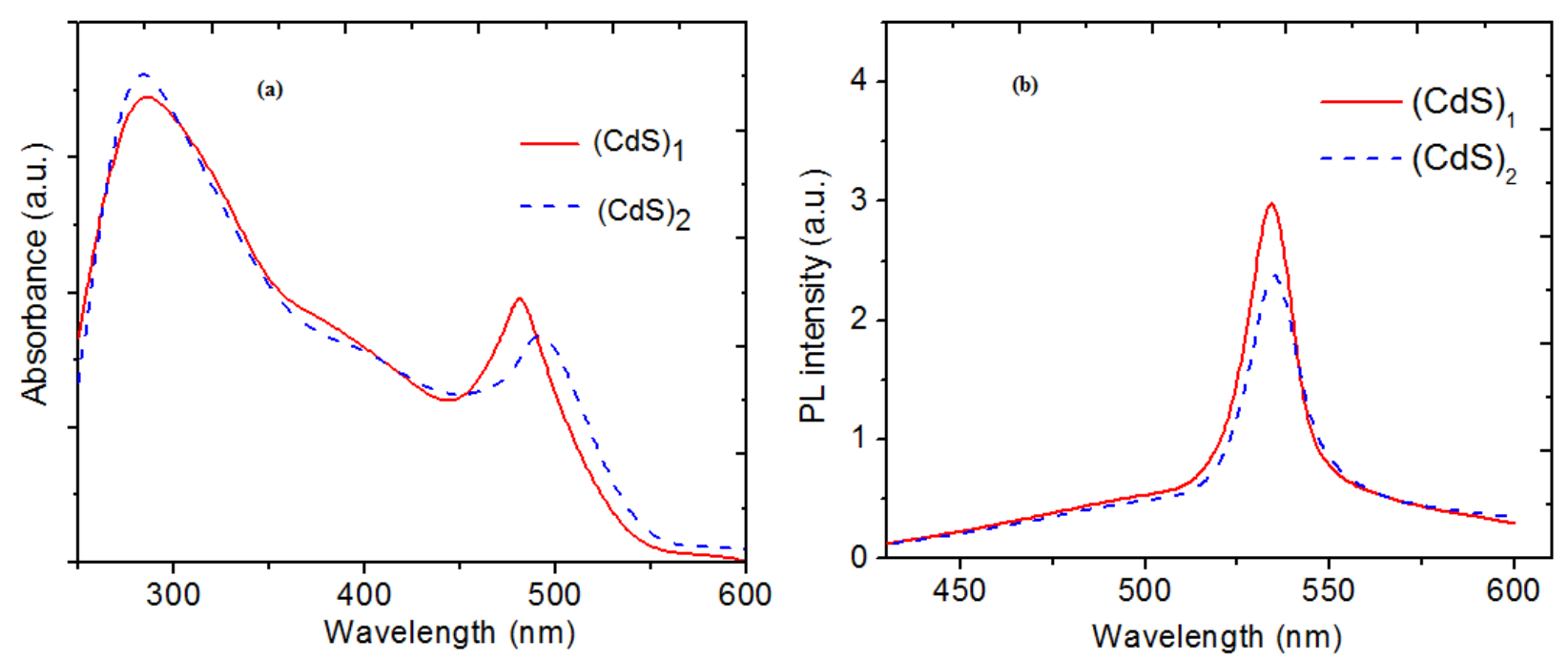

Fig. 3. (a) Optical spectra of CdS nanocrystals (b) Photoluminescence spectra

The UV-Vis absorption spectra of dispersed $(\mathrm{CdS}) 1$ and $(\mathrm{CdS}) 2$ were measured in the region $250-550 \mathrm{~nm}$ at room temperature and shown in Fig. 3a. The optical spectra can be used to calculate an approximate direct band gap using the Tauc relation [15].

$$
\alpha=A\left(h v-E_{\mathrm{g}}\right) / 2 h v
$$

Where $\alpha$ is absorption coefficient, $E \mathrm{~g}$ is the absorption band gap, and $A$ is a constant.

Hexagonal (CdS)1 and (CdS)2 have optical band gaps of $2.52 \mathrm{eV}(484 \mathrm{~nm})$ and $2.48 \mathrm{eV}(496 \mathrm{~nm})$, respectively, at room temperature. The band edges for both the samples are blue-shifted in relation to the bulk material [16]. 
The particle size of nanocrystals is also calculated using Brus equation [17]-

$E^{\text {eff }}=E_{g}+\frac{h^{2} \pi^{2}}{2 \mu R^{2}}-\frac{1.8 e^{2}}{4 \pi \varepsilon \varepsilon_{0} R}$

And $\left(1 / \mu=1 / m_{*}^{*}+1 / m_{h}^{*}\right)$, where $m_{e}^{*}$ is the effective mass of the electron $(0.19 \mathrm{me}), \mathrm{m}_{\mathrm{h}} *$ is the effective mass of hole $(0.8 \mathrm{mh}), \mathrm{R}$ is the radius of the particle, e is the dielectric constant $(5.7)$ and $\varepsilon_{0}$ is the permittivity of free space. The particle size of the CdS nanoparticles as estimated using the above equation are found to be $39 \mathrm{~nm}$ and $48 \mathrm{~nm}$ for $(\mathrm{CdS})_{1}$ and $(\mathrm{CdS})_{2}$, nearly similar to that obtained from the XRD and TEM analysis.

Fig. $3 \mathrm{~b}$ shows photoluminescence spectra of nanocrystals. Bulk CdS is reported to have a broad emission with the emission maximum in the $500-700 \mathrm{~nm}$ region of the luminescence spectrum. The emission is due to recombination from surface defects, redominantly sulfur vacancies $\left(\mathrm{V}_{\mathrm{S}} / \mathrm{V}_{\mathrm{S}}\right)$. The photoluminescence spectra of $(\mathrm{CdS}) 1$ and $(\mathrm{CdS}) 2$ are red-shifted in relation to the corresponding absorption spectra, exhibiting their emission maxima at 532 and $534 \mathrm{~nm}$, respectively. The Stokes shifted band edge luminescence has been attributed to emission from a "dark exciton". It has been observed that the nature of the emitting states change as the cluster size decreases, and the luminescence intensity band gap and the number of surface states increases [18].

The electrical conductivity ( $\sigma \mathrm{dc}$ and $\sigma \mathrm{ac}$ ) of CdS nanocrystals as a function of temperature is shown in Fig. 4a. The variation of $\log \sigma d c$ with temperature $T(K)$ shows that there are two distinct temperature zones with two characteristic regions. The first region from room temperature up to $475 \mathrm{~K}$ that indicating a slow increase of dc conductivity $(\sigma \mathrm{dc})$ with temperature and the second region above $475 \mathrm{~K}$, indicating a rapid increase in dc conductivity. The ac conductivity ( $\sigma \mathrm{ac}$ ) of $\mathrm{CdS}$ nanocrystalsis found to be increases slowly in the temperature range between room temperature up to $400 \mathrm{~K}$ and above this temperature increased rapidly up to $450 \mathrm{~K}$. 

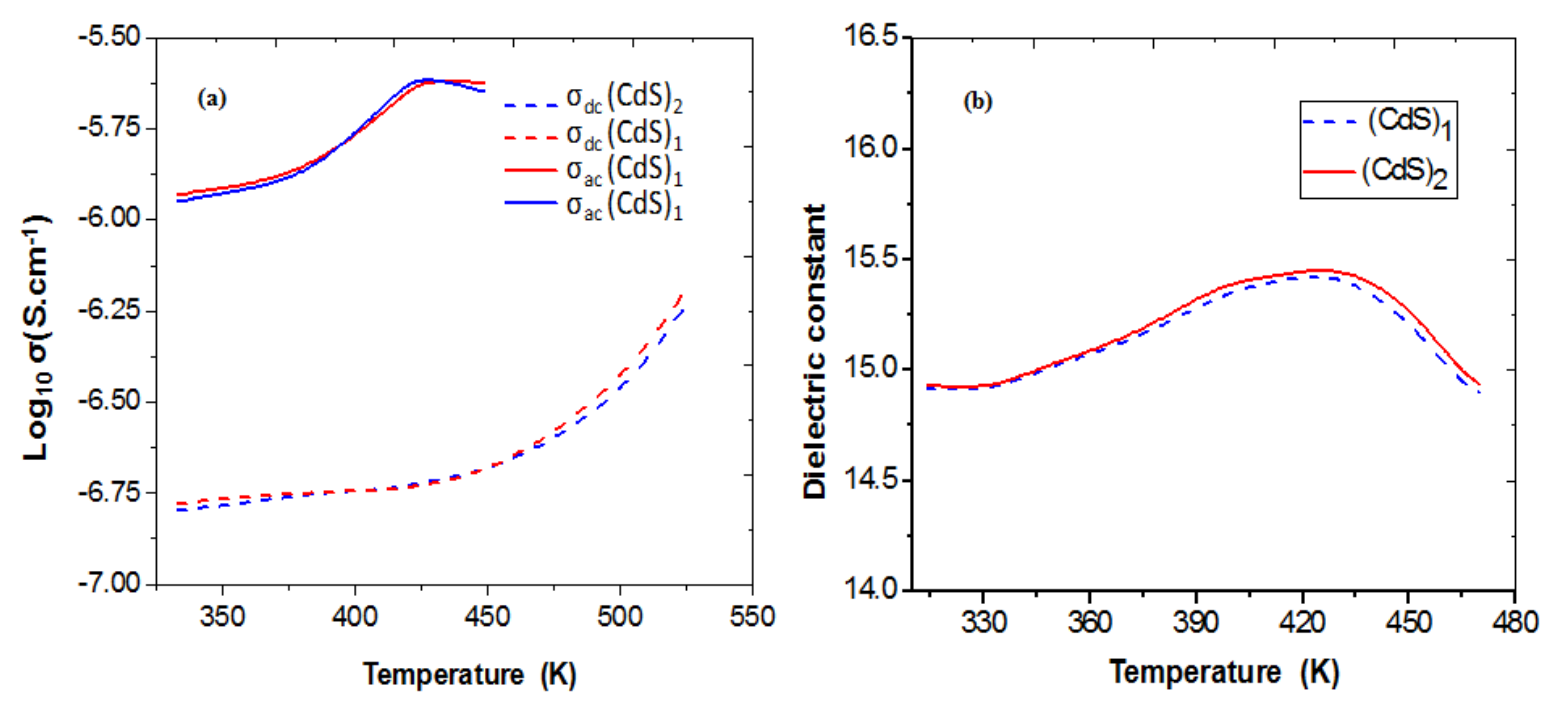

Fig. 4. (a) Variation of ac and dc conductivity with temperature (b) Temperature dependence of dielectric constant.

The CdS nanocrystals show a maximum around almost the same oac above $450 \mathrm{~K}$ temperature, it may be attributed to the large band gap (nanosize), which means that the charges are not released from the particles by thermionic emission and are therefore not available for tunneling [19]. It is seen that the plot of the $\sigma \mathrm{dc} \mathrm{v} / \mathrm{s} \mathrm{T}(\mathrm{K})$ is almost a straight line at lower temperature range up to $475 \mathrm{~K}$, showing an Arrhenius behavior in which the electrical conductivity is proportional to $\exp (\Delta \mathrm{Ea} / \mathrm{KT})$, where $\Delta \mathrm{Ea}$ is the activation energy of the electrical conduction. The activation energy has been determined using slop of the plot between $\ln (\sigma \mathrm{dc})$ and $\mathrm{T}(\mathrm{K})$. The activation energy value of $(\mathrm{CdS})_{1}$ and $(\mathrm{CdS})_{2}$ nanocrystals have been found to be $0.64 \mathrm{ev}$ and $0.70 \mathrm{eV}$ respectively. All the physicochemical properties of the prepraed CdS naocrystals are tabulated in Table- 1 . Because of the small size of the $(\mathrm{CdS})_{1}$ particles, the charge carriers reach the surface of the particles more easily enabling, easy electron transfer by thermionic emission, or tunneling or both, and resulting in a slightly enhanced conductivity compared to that of $(\mathrm{CdS})_{2}$. The dielectric constant values of CdS nanoparticles are found to be larger than the corresponding values for bulk $\mathrm{CdS}$ crystals [20]. The dielectric constant $(\mathcal{C})$ of $(\mathrm{CdS})_{1}$ nanoparticles was slightly greater than that of $(\mathrm{CdS})_{2}$. Also бac of CdS1 is slightly larger than that of the $(\mathrm{CdS})_{2}$ crystals as shown in Fig. $4 \mathrm{~b}$. 
Table 1: Physicochemical properties of CdS naocrystals.

\begin{tabular}{cccccc}
\hline Abbreviations & $\begin{array}{c}\text { Size } \\
(\mathrm{nm})\end{array}$ & $\begin{array}{c}\text { Electronic spectra } \\
(\lambda \max \text { in } \mathrm{nm})\end{array}$ & $\begin{array}{c}\text { Band gap } \\
(\mathrm{eV})\end{array}$ & $\begin{array}{c}\text { PL spectra } \\
(\lambda \max \text { in } \mathrm{nm})\end{array}$ & $\begin{array}{c}\text { Activation } \\
\text { energy }(\mathrm{eV})\end{array}$ \\
\hline$(\mathrm{CdS})_{1}$ & 40 & 484 & 2.52 & 532 & 0.64 \\
$(\mathrm{CdS})_{2}$ & 50 & 496 & 2.48 & 534 & 0.72 \\
\hline
\end{tabular}

\section{Conclusion}

Pure single hexagonal CdS nanocrystals were synthesized by hydrothermal treatment of cadmium thiosemicarbazide complexes. By changing the temperature of the system, different size nanoparticles were obtained. The sizes of $(\mathrm{CdS})_{1}$ and $(\mathrm{CdS})_{2}$ were 40 and $50 \mathrm{~nm}$ respectively and their morphologies were similar. The optical and electrical properties of the CdS nanocrystals show extraordinary behavior compared to that of the bulk CdS crystals. The optical band gap energies $(2.52 \mathrm{eV} / 2.48 \mathrm{eV})$ and photoluminescence peaks $(532 / 534 \mathrm{~nm})$ of the synthesized CdS nanocrystals suggest that they may be promising photocatalysts. The conductivities and the dielectric constants of the CdS nanocrystals were also characterized. The activation energy value of $(\mathrm{CdS})_{1}$ and $(\mathrm{CdS})_{2}$ nanocrystals have been found to be $0.64 \mathrm{eV}$ and 0.70 eV respectively.

\section{Acknowledgements}

This work is supported by Deanship of Scientific Research, Research Center, College of Science, King Saud University, Riyadh, Kingdom of Saudi Arabia. 


\section{References}

[1] A.P. Alivisatos, Semiconductor Clusters, Nanocrystals, and Quantum Dots. Science 271 (1996) 933-937.

[2] Q. Lu,, F. Gao, S. Komarneni, Biomolecule-Assisted Synthesis of Highly Ordered Snowflakelike Structures of Bismuth Sulfide Nanorods. J. Am. Chem. Soc. 126 (2004) 54-55.

[3] X.Y. Chen; X. Wang; Z.H. Wang; X.G. Yang; Y.T. Qian, Hierarchical growth and shape evolution of $\mathrm{HgS}$ dendrites. Cryst. Growth Des., 5 (2005) 347-350.

[4] W. Qingqing, X. Gang, H. Gaorong, Solvothermal synthesis and characterization of uniform CdS nanowires in high yield. J. Solid State Chem.,178 (2005) 680-2685.

[5] G. Shen, C.J. Lee, CdS multipod-based structures through a thermal evaporation processJ. Cryst. Growth Des.5 (2005) 1085-1089.

[6] F. Gao, Q. Lu, S Xie, D.A. Zhao, A Simple Route for the Synthesis of Multi-Armed CdS Nanorod-Based Materials. Adv. Mater. 14 (2002)1537-1540.

[7] Y.D. Yin, A.P. Alivisatos. Colloidal nanocrystal synthesis and the organic-inorganic interface. Nature.437 (2005) 664-670.

[8] K.T. Thurn, E.M.B. Brown, A. Wu, Nanoparticles for Applications in Cellular Imaging. Nanoscale Res. Lett. 2(2007) 430-441.

[9] P.A. Ajibade, D.C. Onwudiwe, M.J. Moloto, Synthesis of hexadecylamine capped nanoparticles using group 12 complexes of N-alkyl-N-phenyl dithiocarbamate as singlesource precursors, Polyhedron, 30 (2011) 246-252.

[10] P. Shadma, T. Ahamad, N. Nishat, New anti-bacterial polychelates: synthesis, characterization, and anti-bacterial activities of thiosemicarbazide-formaldehyde resin and its polymer-metal complexes, Appl. Organometal. Chem., 22 (2008) 70-77.

[11] Z.L. Wang, Particle \& Particle Systems Characterization, Wiley Inter Science, 18 (2001) 142-165. 
[12] X.Y. Chen, Z.H. Wang, X. Wang, R. Zhang, X.Y. Liu, W.J. Lin, Y.T. Qian, J.Cryst. Growth, 263 (2004) 570-574.

[13] S. Gorai. D. Ganguli, S. Chaudhuri, Mater. Lett, 59 (2005) 826-828.

[14] J. Tauc, A. Menth, States in the gap, J. Non-Cryst. Solids 8-(10) (1972) 569-73.

[15] I.J. Pankove. Optical Processes in Semiconductors. New York: Dover Publications Inc; 1971.

[16] Z.R. Khan, M. Zulfequar, M.S. Khan, Chemical synthesis of CdS nanoparticles and their optical and dielectric studies. J. Mater. Sci., 46 (2011) 5412-5416.

[17] A.L. Efros, M. Rosen, M. Kuno, M. Nirmal, D.J. Norris, M.G. Bawendi. Band-edge exciton in quantum dots of semiconductors with a degenerate valence band: dark and bright exciton states. Physical Review B: Condensed Matter, 54 (1996) 4843-4856.

[18] J.H. Fendler, Atomic and molecular clusters in membrane mimetic chemistry. Chem. Rev. 87 (1989) 877-899.

[19]K. K.Nanda, S. N. Sarangi, and S. N. Sahu. Phys. Sem. Nanostruct. 217 (1997).

[20]Z.R. Khan, M. Zulfequar, M.S. Khan, Optical and structural properties of thermally evaporated cadmium sulphide thin films on silicon (100) wafers, Mater. Sci. Eng B, 174 (2010) 145. 